\title{
SOME CHARACTERIZATIONS OF SYSTEMS OF LINEAR DIFFERENTIAL EQUATIONS HAVING REGULAR SINGULAR SOLUTIONS( $\left.{ }^{1}\right)$
}

\author{
BY \\ DONALD A. LUTZ
}

I. Introduction. In this paper, we consider the system of $n$ linear ordinary differential equations

$$
X^{\prime}(z)=\left(z^{-q} \sum_{v=0}^{\infty} A_{v} z^{v}\right) X(z)=A(z) X(z),
$$

where $q$ is an integer, and the $A_{v}$ are constant $n \times n$ matrices for which the series converges componentwise in some neighborhood of zero.

We will investigate structural properties induced on $A(z)$ under the assumption that the solutions to (1.1) have the regular singular behavior near 0 . This behavior may be characterized, for example, by the requirement that all solution vectors to (1.1) have components which are finite sums of products of analytic functions of $z$ at 0 , complex powers of $z$, and integer powers of $\log z$. Systems (1.1), all of whose solutions have regular singular behavior, will be called regular singular systems.

Fundamental solution matrices for such systems can be represented as

$$
\Phi(z)=S(z) z^{R}
$$

in a neighborhood of 0 , where $S(z)$ is an invertible matrix of single-valued analytic functions and $R$ is a constant $n \times n$ matrix.

If $q \leqq 0$, then 0 is a regular point of (1.1), so every fundamental solution has the representation (1.2) with $R=0$. If $q=1$, then a classical theorem of M. L. Sauvage states that every fundamental solution has the form (1.2). (For a modern proof for this theorem, see [1, pp. 73-74].) Therefore when $q \leqq 1, A(z)$ has no further restrictions, so we will assume henceforth that $q \geqq 2$.

In a recent note [2], the author has shown that the $n$ symmetric functions of $A(z)$ have poles at 0 whose order is somewhat restricted in case $A(z)$ corresponds to a regular singular system. These conditions force the first $q-1$ coefficient matrices in the expansion of $A(z)$, i.e., the principal part of $A(z)$, to have a special structure. In particular, for $A_{0}$ and $A_{1}$ we have the rather complete information that

(i) $A_{0}$ must be nilpotent, and

(ii) $\operatorname{tr} A_{0}^{k} A_{1}=0$ for each $k=0,1,2, \ldots, n-1$, provided $q>2$.

Presented to the Society, September 1, 1965 under the title Regular-singular systems of linear differential equations; received by the editors February 4, 1966.

(1) This appeared as part of the author's doctoral dissertation completed at Syracuse University and Aachen, Germany under the direction of Professor W. B. Jurkat. 
But neither the conditions (i) and (ii) nor the conditions on the principal part of $A(z)$, although sharp, come close to being also sufficient. We may ask, however, how many of the matrices $A_{v}$ in the expansion of $A(z)$ are involved in necessary and sufficient conditions. This is equivalent to the following problem:

If $A(z)$ is an $n \times n$ matrix having a pole of order $q \geqq 2$, and if $B(z)$ is an $n \times n$ matrix of functions which are meromorphic at 0 , then what order singularity for $B(z)$ guarantees that the perturbed equation

$$
X^{\prime}(z)=(A(z)+B(z)) X(z)
$$

is regular singular simultaneously with (1.1)?

The following theorem answers this question.

THEOREM 1. If $B(z)$ is an $n \times n$ matrix having a zero of at least the order $(n-1) q-n$, then (1.1) is regular singular if and only if (1.3) is.

Restating this, we can tell how many coefficients of $\sum A_{v} z^{v}$ are involved in determining regular singular behavior.

COROLlaRY 1.1. If $(A)_{m}$ denotes the truncation of the Laurent expansion for $A(z)$ after the mth term, then (1.1) and $X^{\prime}=(A)_{m} X$ are regular singular simultaneously in case $m \geqq n(q-1)$.

This bound will be shown to be exact (§IV) for a certain class of matrices.

But we may still persist in asking for the necessary and sufficient conditions. If $A(z)$ were a companion matrix, then (1.1) would be equivalent to an $n$th order linear differential equation. In this case L. Fuchs found that the necessary and sufficient conditions can be phrased in an exceptionally simple way in terms of bounds on only the order of the poles of the $n$ symmetric functions of $A(z)$ at 0 . For systems not of this form, there appears to be no correspondingly simple criteria.

One rather pleasing answer to the problem of representation of these conditions was found by W. B. Jurkat and orally communicated to the author.

THEOREM A (JURKAT). With A(z), we associate a sequence of matrices $\left\{\mathfrak{A}_{v}\right\}$, inductively defined by

$$
\mathfrak{A}_{v+1}=\mathfrak{A}_{v}^{\prime}+\mathfrak{A}_{v} A ; \quad \mathfrak{U}_{0}=I .
$$

Let $p\left[\mathfrak{A}_{v}\right]$ denote the order of the pole of $\mathfrak{A}_{v}$ at 0 . Then (1.1) is regular singular at 0 if and only if

$$
p\left[\mathfrak{Q}_{v}\right] \leqq v+M
$$

for all $v=0,1,2, \ldots$, where $M$ is a fixed constant, independent of $v$.

We are able to find the exact value for $M$ in case we take into account only the dimension of the system and the order of the pole of $A(z)$. In our proof, it is also apparent that the conditions need only be checked for a finite number of $v$. 
THEOREM 2. (1.1) is regular singular at 0 if and only if

$$
p\left[\mathfrak{A}_{v}\right] \leqq v+(n-1)(q-1)
$$

for all $v=n, n+1, \ldots, N$, where $N$ is a constant depending upon $A(z)$.

In case the leading coefficient matrix $A_{0}$ is nilpotent of degree $n$, i.e., it has full rank, we are able to estimate $N(\S \mathrm{VI})$.

Since the coefficients in the expansion of $\mathfrak{A}_{v}$ are homogeneous combinations of the matrices $A_{v}$, the following corollary gives an answer to the question of representation of conditions.

COROLLARY 2.1. There exist a finite number of homogeneous polynomial identities which the first $n(q-1)$ coefficients of $A(z)$ must satisfy if and only if $(1.1)$ is regular singular. The number of identities is dependent upon properties of $A(z)$.

We remark now that throughout the remainder of this paper, we establish the convention that a zero of the order $h<0$ means a pole of the order $|h|$.

II. Three lemmas. It has been shown by the author [2] and in several earlier works (see, for example [1, pp. 74-75]) that the regular singular property for (1.1) at 0 is equivalent to the existence of a substitution $X(z)=T(z) Y(z)$ with the following properties:

(2.1) $T(z)=P(z) D(z)$, where $P(z)$ and $P^{-1}(z)$ are $n \times n$ matrices of functions analytic at 0 ,

(2.2) $D(z)=\operatorname{diag}\left\{z^{\alpha_{1}}, z^{\alpha_{2}}, \ldots, z^{\alpha_{n}}\right\}, \alpha_{1}, \ldots, \alpha_{n}$ are integers, and

(2.3) $T^{-1}(z) A(z) T(z)-T^{-1}(z) T^{\prime}(z)=C(z)$, the coefficient matrix for the system corresponding to $Y(z)$, has a pole at 0 whose order is at most one.

J. Horn [3] first investigated transformations of this type (although not in the factorization (2.1)). If $T(z)$ is an $n \times n$ matrix of functions meromorphic in some neighborhood of 0 , det $T(z) \not \equiv 0$, and if $T(z)$ satisfies property (2.3), we will say that $T(z)$ is a transformation of $A(z)$ of Horn's type. We denote the totality of all such transformations by $H(A)$. Then (1.1) is regular singular at 0 if and only if $H(A)$ is nonempty.

Given $T \in H(A), T$ can be factored in its Smith Normal Form [4, p. 41] as $T(z)=P(z) D(z) Q(z)$, where $P, P^{-1}, Q$, and $Q^{-1}$ are convergent power series, $D$ is as in (2.2) and, moreover, the integers $\alpha_{i}$ are uniquely determined from $T$ when monotonically ordered as $\alpha_{1} \leqq \alpha_{2} \leqq \cdots \leqq \alpha_{n}$. (See [5, p. 384].) $T(z)$ can then be modified to $T(z) Q^{-1}(z)$ and $T \in H(A)$ if and only if $T Q^{-1} \in H(A)$. We will usually assume henceforth that we have the later simplified structure (2.1).

The power series $P(z)$ transforms $A(z)$ into

$$
\hat{A}(z)=P^{-1}(z) A(z) P(z)-P^{-1}(z) P^{\prime}(z),
$$

which has a pole of order $q$ and has the property that $D \in H(\hat{A})$ if and only if $T \in H(A)$. 
The maximal difference, $\alpha_{n}-\alpha_{1}$, of the exponents determines to a great extent the structure of $T$ and will be useful in the examination of the structure of $A$. After Moser [4, p. 386] we define the maximal difference to be the span of $T$ and denote it by $s(T)$. We remark that the span is defined only for matrices $T$ whose inverse exists and, moreover, $s(T)=s\left(T^{-1}\right)$.

If $T \in H(A)$, then unfortunately $s(T)$ is not uniquely determined in terms of properties of $A(z)$, in fact, if $A=A_{0} / z^{q}$ when $A_{0}$ is nilpotent, then there exists $T_{1} \in H(A)$ such that $s\left(T_{1}\right)$ is larger than any preassigned natural number.

For the applications which we have in mind, some upper bound on the span is desirable, but we do not need this upper bound on the spans of all $T \in H(A)$ (which, by the above example, is impossible anyway). A bound on the span of one transformation in $H(A)$ proves to be sufficient. Such a transformation and bound are now constructed in the following lemma.

LEMMA 1. If (1.1) is regular singular at 0 , there exists $\hat{T} \in H(A)$ such that $s(\hat{T}) \leqq(n-1)(q-1)$.

Proof. Let $\hat{D}=\operatorname{diag}\left\{z^{x_{1}}, z^{x_{2}}, \ldots, z^{x_{n}}\right\}$, where $x_{1} \leqq x_{2} \leqq \cdots \leqq x_{n}$. If we define $\hat{T}=P \hat{D}$, where $P$ is from (2.1) and (2.4), then $\hat{D} \in H(\hat{A})$ if and only if

$$
\hat{D}^{-1} \hat{A} \hat{D}
$$

has a pole whose order is at most one. If $\hat{A}=\left(\hat{a}_{i j}\right)_{1}^{n}$ and if we let $-p_{i j} \leqq q$ denote the order of the pole of $\hat{a}_{i j}$, then (2.5) has at worst a simple pole if and only if the system of $n^{2}$ linear inequalities

$$
x_{j}-x_{i} \geqq-1-p_{i j}, \quad 1 \leqq i, j \leqq n
$$

is satisfied by a vector $x=\left(x_{1}, x_{2}, \ldots, x_{n}\right)$. Our problem now is to estimate from above the quantity $x_{n}-x_{1}$ when $x$ is a solution to (2.6) and the components of $x$ are ordered monotonically.

We first remark that the existence of $T=P D$, where $D$ has the form (2.2) and $T$ satisfies (2.3), means that $\alpha=\left(\alpha_{1}, \alpha_{2}, \ldots, \alpha_{n}\right)$ is a solution to (2.6). We will achieve our solution by comparing this unknown solution $\alpha$ with a sequence of other solutions to (2.6).

Without any loss in generality, we may take $x_{1}=\alpha_{1}=0$, since only differences of the components appear in the system (2.6).

Let $k$ be a fixed integer, $1 \leqq k \leqq n$. We wish to show the existence of

$$
\lambda=\left(\lambda_{1}, \lambda_{2}, \ldots, \lambda_{n}\right)
$$

such that

(1) $0=\lambda_{1} \leqq \lambda_{2} \leqq \cdots \leqq \lambda_{n}$,

(2) $\lambda$ is a solution to (2.6), and

(3) $\lambda_{k} \leqq(k-1)(q-1)$. 
This will be proven using an induction on $k$. For $k=1$, we take $\lambda=\alpha$, in which case (1) and (2) follow from (2.2) and (2.3) and (3) is trivially true. We assume (1)-(3) for $k$ and seek to prove them for $k+1$. To this end we define

$$
\lambda_{k+1}^{*}=\min \left(\lambda_{k+1}-\lambda_{k}, q-1\right)+\lambda_{k}
$$

and form the $n$-tuple $\lambda^{*}=\left(\lambda_{1}^{*}, \ldots, \lambda_{n}^{*}\right)$, where

$$
\begin{aligned}
\lambda_{i}^{*} & =\lambda_{i} & & \text { if } 1 \leqq i \leqq k, \\
& =\lambda_{i}-\lambda_{k+1}+\lambda_{k+1}^{*} & & \text { if } k+1 \leqq i \leqq n .
\end{aligned}
$$

We claim that

(1') $0=\lambda_{1}^{*} \leqq \lambda_{2}^{*} \leqq \cdots \leqq \lambda_{n}^{*}$,

(2) $\lambda^{*}$ is a solution to (2.6), and

(3') $\lambda_{k+1}^{*} \leqq k(q-1)$.

To prove (1') we note that $\lambda_{i+1}^{*}-\lambda_{i}^{*}=\lambda_{i+1}-\lambda_{i} \geqq 0$ from (1) in case $i \leqq k-1$ or $i \geqq k+1$, and

$$
\lambda_{k+1}^{*}-\lambda_{k}^{*}=\lambda_{k+1}^{*}-\lambda_{k}=\min \left(\lambda_{k+1}-\lambda_{k}, q-1\right) \geqq 0 .
$$

Since $\lambda_{i}^{*}-\lambda_{j}^{*}=\lambda_{i}-\lambda_{j}$ unless

(I) $i \leqq k$ and $j \geqq k+1$, or else

(II) $i \geqq k+1$ and $j \leqq k$,

we need only check these cases in order to verify $\left(2^{\prime}\right)$.

Case I.

$$
\lambda_{i}^{*}-\lambda_{j}^{*}=\lambda_{i}-\lambda_{j}+\lambda_{k+1}-\lambda_{k+1}^{*} \geqq-1-p_{j i}+\left(\lambda_{k+1}-\lambda_{k+1}^{*}\right) \geqq-1-p_{j i}
$$

since $\left(\lambda_{k+1}-\lambda_{k+1}^{*}\right) \geqq 0$.

Case II.

$$
\lambda_{i}^{*}-\lambda_{j}^{*}=\lambda_{i}-\lambda_{k+1}+\lambda_{k+1}^{*}-\lambda_{j}=\lambda_{i}-\lambda_{j} \geqq-1-p_{j i}
$$

in case $\lambda_{k+1}^{*}=\lambda_{k+1}$, and

$$
\lambda_{i}^{*}-\lambda_{j}^{*}=q-1+\lambda_{k}+\lambda_{i}-\lambda_{k+1}-\lambda_{j} \geqq-1-p_{j i}+\left(\lambda_{k}-\lambda_{j}\right)+\left(\lambda_{i}-\lambda_{k+1}\right) \geqq-1-p_{j i}
$$

otherwise, since both $\left(\lambda_{k}-\lambda_{j}\right)$ and $\left(\lambda_{i}-\lambda_{k+1}\right)$ are nonnegative. Finally we have $\lambda_{k+1}^{*} \leqq q-1+\lambda_{k} \leqq k(q-1)$ and the induction is completed. Taking $k=n$, we have an $n$-tuple satisfying our requirements, and hence Lemma 1 is proven.

The bound achieved on $s(T)$ is exact in case we consider systems whose leading coefficient matrix has full rank. If $A(z)=A_{0} / z^{q}$, where $A_{0}$ is nilpotent of full rank, then for each $T \in H(A), s(T) \geqq(n-1)(q-1)$ and so our minimal span is exactly the lower bound also.

In case $A_{0}=H_{1} \oplus H_{2} \oplus \cdots \oplus H_{s}$, where each $H_{i}$ is nilpotent of full rank, then the bound on $s(T)$ for some $T \in H(A)$ can be slightly improved. Since $A_{0}$ is similar to $\hat{A}_{0}$, the leading coefficient matrix of $\hat{A}$, we can replace $q-1$ by $q-2$ in the definition (2.7) in exactly $s-1$ cases, therefore

$$
s(T) \leqq(q-2)(s-1)+(n-s)(q-1)=(n-1)(q-1)-s+1 .
$$


The importance attached to the upper bound on the span of a transformation is now explained by the following lemma, which allows estimation of the order of the poles (or zeros) which can occur in expressions involving $T, T^{-1}$ and their derivatives.

LEMMA 2. If $s(T) \leqq N$ and $B$ has a pole whose order is at most $r$, then $T^{(m)} B T^{-1}$ has a pole whose order is at most $N+m+r$.

Proof. Since it is easier to apply and no more work to prove, we represent $T$ in its general factorization

$$
T(z)=P(z) D(z) Q(z),
$$

where $D(z)$ has the form (2.2) and $P(z)$ and $Q(z)$ are units in the ring $\mathscr{R}$ of $n \times n$ matrices whose elements are analytic functions in a neighborhood of 0 , i.e., the leading coefficient matrix in the expansions of $P$ and $Q$ are invertible. Then differentiating (2.8) we obtain

$$
T^{(m)}=\sum_{i+j+k=m} c_{i j k} P^{(i)} D^{(j)} Q^{(k)}, \quad i, j, k \geqq 0,
$$

where the $c_{i j k}$ are nonzero constants. We compute then

$$
T^{(m)} B T=\sum_{i+j+k=m} c_{i j k} P^{(i)} D^{(j)} Q^{(k)} B Q^{-1} D^{-1} P^{-1} .
$$

Letting $Q^{(k)} B Q^{-1}=B_{k}^{*}=\left(b_{u v k}^{*}\right)_{1}^{n}$, we note that $B_{k}^{*}$ has a pole whose order is at most r. Also

$$
D^{(j)}=D^{*} \operatorname{diag}\left\{z^{\alpha_{1}-j}, \ldots, z^{\alpha_{n}-j}\right\}
$$

where $D^{*}$ is an $n \times n$ constant diagonal matrix, possibly having some zeros on the diagonal too, in case the differentiation annihilates some positive powers of $z$. Then

$$
D^{(j)} B_{k}^{*} D^{-1}=D^{*} B_{k}^{* *}, \quad \text { where } B_{k}^{* *}=\left(b_{u v k}^{*} z^{\alpha_{u}-\alpha_{v}-j}\right)_{1}^{n},
$$

and has a pole whose order is at most $N+j+r$. Equality occurs in case the entry in the $(1, n)$ position of $B_{k}^{*}$ has exactly a pole of order $r$. Since $D^{*}, P^{-1}$, and $P^{(i)}$ are all in $\mathscr{R}$, multiplication by these matrices does not give rise to poles of any higher order. So for each triple $(i, j, k)$,

$$
c_{i j k} P^{(i)} D^{(j)} Q^{(k)} B Q^{-1} D^{-1} P^{-1}
$$

has a pole whose order is at most $N+j+r$. Then $T^{(m)} B T^{-1}$ has a pole whose order is at most the maximum of the orders of the pole of each term in (2.9), and therefore the order does not exceed $N+m+r$.

Lemma 2 has an analogy in case $T$ and $B$ are not meromorphic, but are expansible in Laurent series in the variable $z^{1 / p}$, where $p$ is a positive integer. We first 
generalize the notion of span by considering the reduction to Smith Normal Form relative to the ring $\mathscr{R}^{*}$ of matrices whose elements are convergent power series in $z^{1 / p}$. The notion of a pole of order $q$ must be replaced by the notion that the Laurent expansion in $z^{1 / p}$ begins with $z$ to the rational power $q / p$. We state the corresponding result as

LEMMA 3. Let $T$ have span $\left(r_{n}-r_{1}\right) / p$ and let $B$ have a Laurent series in $z^{1 / p}$ whose leading term contains $z$ to the power $-N / p$. If the mth formal derivative of $T$ is denoted $T^{(m)}$, then $T^{(m)} B T^{-1}$ has a Laurent series in $z^{1 / p}$ whose leading term contains $z$ to the power $r \geqq-N / p-\left(r_{n}-r_{1}\right) / p-m$.

Proof. Let $T(z)=P(z) D(z) Q(z)$, where

$$
D(z)=\operatorname{diag}\left\{z^{r_{1} / p}, \ldots, z^{r_{n} / p}\right\},
$$

be the representation of $T$ in its Smith Normal Form. We perform the change of variable $w=z^{1 / p}$. Then $T\left(W^{p}\right)=P\left(W^{p}\right) D\left(W^{p}\right) Q\left(W^{p}\right)=T^{*}(W)$ has span $\left(r_{n}-r_{1}\right)$ in $W$ since $D\left(W^{p}\right)=\operatorname{diag}\left\{W^{r_{1}}, \ldots, W^{r_{n}}\right\}$ and $P$ and $Q$ are units in $\mathscr{R}$. If

$$
B(z)=\sum_{v=-N}^{\infty} B_{v} z^{v / p},
$$

then

$$
B^{*}(W)=B\left(W^{p}\right)=\sum_{v=-N}^{\infty} B_{v} z^{v}
$$

has a pole of order $N$ in $W$. Using Lemma 2, we conclude that

$$
T^{*}(W)^{(i)} B^{*}(W) T^{*}(W)^{-1}
$$

has a pole in $W$ whose order does not exceed $i+N+\left(r_{n}-r_{n}\right)$. But

$$
\frac{d^{m}}{d\left(W^{p}\right)^{m}} T\left(W^{p}\right)=\sum_{i=1}^{m} C_{i} W^{i-m-m(p-1)} \frac{d^{i}}{d W^{i}} T^{*}(W)
$$

where $C_{i}$ are constants. Therefore

$$
T^{(m)}(z) B(z) T^{-1}(z)=\sum_{i=1}^{m} C_{i} W^{i-m-m(p-1)} \frac{d^{i}}{d W^{i}} T^{*}(W) B^{*}(W) T^{*}(W)^{-1}
$$

The general term in the sum on the right-hand side of (2.10) has a pole in $W$ whose order does not exceed $i+N+\left(r_{n}-r_{1}\right)+m(p-1)+m-i$, hence the right-hand side of (2.10) has a pole in $W$ whose order does not exceed $N+\left(r_{n}-r_{1}\right)+m p$. Therefore $T^{(m)}(z) B(z) T^{-1}(z)$ has a Laurent series in $z^{1 / p}$ whose leading term contains $z$ to no smaller power than

$$
-\frac{N}{p}-\frac{\left(r_{n}-r_{1}\right)}{p}-m
$$


III. Proof of Theorem 1. Assume (1.1) is regular singular. Then according to Lemma 1 , there exists $T \in H(A)$ such that $s(T) \leqq(n-1)(q-1)$. But $T \in H(A+B)$ also, since

$$
T^{-1}(A+B) T-T^{-1} T^{\prime}=C+T^{-1} B T
$$

and if we apply Lemma 2 with $T$ replaced by $T^{-1}$, we find that $T^{-1} B T$ has a pole whose order does not exceed

$$
(n-1)(q-1)-[(n-1) q-n]=1 .
$$

Therefore (1.3) is regular singular.

Conversely, assume (1.3) is regular singular. Then Lemma 1 provides us with a $U \in H(A+B)$ such that $s(U) \leqq(n-1)(q-1)$. Likewise $U \in H(A)$ by the same argument as above; hence (1.1) is regular singular and the proof of Theorem 1 is completed.

IV. An example showing the bound in Corollary 1.1 to be exact. Let $A_{0}$ be a nilpotent matrix of order $n$ which has full rank. Assume $A_{0}$ is in its Jordan canonical form

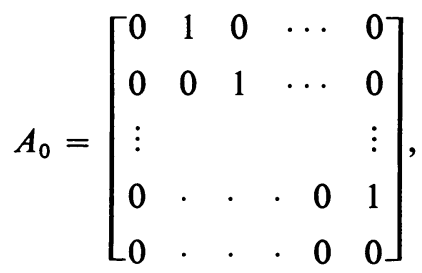

and let

$$
A(z)=A_{0} / z^{q}+R(z)
$$

where $R(z)$ has all entries equal to zero except the function $z^{(n-1) q-n-1}$ in the $(n, 1)$ position and $q \geqq 2$, as usual.

A necessary condition for (1.1), with a matrix in this form, to be regular singular is that the determinant of $A(z)$ have a pole whose order does not exceed $n$. But $\operatorname{det} A(z)=z^{-n-1}$, therefore (1.1) is not regular singular. However,

$$
(A)_{n(q-1)-1}=A_{0} / z^{q}
$$

and obviously

$$
X^{\prime}=A_{0} / z^{q} X
$$

is regular singular. Therefore we may not truncate the Laurent series for $A(z)$ before $n(q-1)$ terms without risking the alteration of the regular singular property.

We present here a sketch of the proof of the necessity of the condition on det $A(z)$ mentioned above. 
$A(z)$ can be transformed into a companion matrix using the substitution $T X=Y$, where

$$
T=\left[\begin{array}{l}
\alpha_{0} \\
\alpha_{1} \\
\vdots \\
\alpha_{n-1}
\end{array}\right],
$$

in which the row-vectors $\alpha_{v}$ are defined by

and

$$
\alpha_{0}=(1,0, \ldots, 0)
$$

$$
\alpha_{v}=\alpha \cdot(\mathscr{D}+A)^{v} .
$$

$\mathscr{D}$ denotes the matrix differential operator $\mathscr{D}=\operatorname{diag}\{d / d z, \ldots, d / d z\}$ and $(\mathscr{D}+A)^{v}$ denotes the $v$ th iterate of the right-hand operator $(\mathscr{D}+A)$.

$T$, so defined, is an invertible matrix since it is lower triangular with no diagonal element being identically zero.

Then necessary and sufficient conditions for (1.1) to be regular singular are that each of the $n$ symmetric functions of $T A T^{-1}+T^{\prime} T^{-1}=T\left(A+T^{-1} T^{\prime}\right) T^{-1}$ have a pole whose order does not exceed the rank of the symmetric function. Therefore it is necessary for det $\left(A+T^{-1} T^{\prime}\right)$ to have at worst a pole of order $n$. But by an elementary computation, it can be shown that $\operatorname{det}\left(A+T^{-1} T^{\prime}\right)=\operatorname{det} A+($ a function having a pole of order $n$ ).

V. Proof of Theorem 2. The ideas used in the proof are similar to those in the proof of Theorem A.

If (1.1) is regular singular, then again from Lemma 1 we have $T \in H(A)$ such that $s(T) \leqq(n-1)(q-1)$. Solving (2.3) for $A$, we find

$$
A=T C T^{-1}+T^{\prime} T^{-1}
$$

The sequences $\left\{\mathfrak{A}_{v}\right\}$ and $\left\{C_{v}\right\}$, associated with $A$ and $C$, respectively and formed as in Definition (1.4), are related by the following identities:

$$
\mathfrak{A}_{v}=\sum_{k=0}^{v}\left(\begin{array}{l}
v \\
k
\end{array}\right) T^{(k)} C_{v-k} T^{-1}, \quad v=0,1,2, \ldots
$$

These can be verified by an easy induction on $v$.

Since $C$ has a pole of at worst the order one, then $C_{v}$ has a pole whose order is at most $v$ since differentiation and multiplication on the right by $C$ can increase the order of the pole by at most one as $v$ increases to $v+1$. We now use Lemma 2 to estimate the order of the pole of terms occurring on the right-hand side of (5.2). $T^{(k)} C_{v-k} T^{-1}$ has a pole whose order does not exceed

$$
v-k+k+s(T) \leqq v+(n-1)(q-1) .
$$

Therefore $p\left[\mathfrak{A}_{v}\right] \leqq \max _{0 \leqq k \leqq v}\left\{p\left[T^{(k)} C_{v-k} T^{-1}\right]\right\} \leqq v+(n-1)(q-1)$ for each $v=0,1$, $2, \ldots$ and the necessity of condition (1.6) is proven. 
For sufficiency, we turn to a theorem of H. L. Turrittin, which demonstrates the existence of a matrix $P(z)$, which transforms $A$ into a canonical form $B(z)$, for which the function $p\left[\mathfrak{B}_{v}\right]$ is easy to control. This matrix $P(z)$ is, however, unfortunately not meromorphic, but has the form

$$
P(z)=\sum_{k=0}^{w} P_{k} z^{k / p}
$$

where $p$ and $W$ are positive integers, $P_{k}$ are constant matrices, and in some deleted neighborhood of 0 , det $P(z) \neq 0$. Turrittin's result $[6$, pp. 42-46] we now state as:

TheOREM B (TurritTIN). Corresponding to the system (1.1), there exists a substitution $X(z)=P(z) Y(z)$, where $P(z)$ has the form (5.3), which reduces (1.1) to the canonical form

$$
Y^{\prime}(t)=\left(\sum_{v=0}^{\infty} B_{v} t^{v-h}\right) Y(t)=B(t) Y(t)
$$

where
(a) $t=z^{1 / p}$,
(b) $B_{0} \neq 0$,
(c) $h$ is a nonnegative integer,
(d) $B_{0}$ is a diagonal matrix with at least one nonzero entry unless $h=0$ or 1 , and
(e) the series converges elementwise in a deleted neighborhood of 0.

We now make the assumption

(I): (1.1) is not regular singular.

Then (5.4) evidently cannot be regular singular either.

Now define

$$
B^{*}(z)=P^{-1}(z) A(z) P(z)-P^{-1}(z) P^{\prime}(z) .
$$

$B^{*}(z)$ is not necessarily meromorphic. It does however have a Laurent expansion in powers of $z^{1 / p}$. We define $p\left[B^{*}(z)\right]$ to be the negative of the smallest exponent of $z$ which occurs in the expansion of $B^{*}(z)$ in powers of $z^{1 / p}$. In case $B^{*}(z)$ would be meromorphic, this coincides with our earlier definition. For each term in the sequence $\left\{\mathfrak{B}_{v}^{*}(z)\right\}$, defined as in (1.4), we define $p\left[\mathfrak{B}_{v}^{*}(z)\right]$ analogously and measure the rate of growth of this function as $v$ increases over the positive integers. However, we note that the function values are no longer integers, but are rational numbers.

The matrices $B(t)$ and $B^{*}(z)$ are related by the change of variable $t=z^{1 / p}$ for the systems

$$
\tilde{Y}^{\prime}(z)=B^{*}(z) \tilde{Y}(z) \text { and (5.4), }
$$

where $Y(t)=\tilde{Y}\left(t^{p}\right)$. Therefore

$$
\begin{aligned}
B^{*}(z) & =\frac{1}{p} z^{-1+1 / p} B\left(z^{1 / p}\right) \\
& =\frac{1}{p} B_{0} z^{-1+(1-h) / p}+(\text { terms containing higher powers of } z) .
\end{aligned}
$$


Then we compute

$$
\mathfrak{B}_{v}^{*}(z)=\frac{1}{p^{v}} B_{0}^{v} z^{v[-1+(1-h) / p]}+(\text { terms containing higher powers of } z),
$$

since the dominating term of the expansion of each member of the sequence always arises from $\mathfrak{B}_{v-1}^{*} B^{*}$. This happens because differentiation increases the order of the leading term by one, while multiplication by $B^{*}$ increases the order by $1+(h-1) / p>1$ since $B_{0}$ is nonnilpotent. Therefore

$$
p\left[\mathfrak{B}_{v}^{*}(z)\right]=v(1+(h-1) / p) \geqq v(1+1 / p),
$$

for all $\grave{v}=0,1,2, \ldots$, since $h \geqq 2$.

From (5.5), we deduce the following identities relating $\left\{\mathfrak{B}_{v}^{*}(z)\right\}$ and $\left\{\mathfrak{A}_{v}\right\}$ :

$$
\mathfrak{B}_{v}^{*}(z)=\sum_{k=0}^{v}\left(\begin{array}{l}
v \\
k
\end{array}\right)\left(P^{-1}\right)^{(k)} \mathfrak{A}_{v-k} P ; \quad v=0,1,2, \ldots
$$

We now make the additional assumption

(II): $p\left[\mathfrak{A}_{v}\right] \leqq v+M$ for all $v=0,1,2, \ldots$, where $M$ is independent of $v$. Then we prove that

$$
p\left[\left(P^{-1}\right)^{(k)} \mathfrak{A}_{v-k} P\right] \leqq v+M_{0}
$$

for all $v=0,1,2, \ldots$, where $M_{0}$ is independent of both $k$ and $v$. Applying Lemma 3 (with $P$ replaced by $P^{-1}$ ), we obtain

$$
p\left[\left(P^{-1}\right)^{(k)} \mathfrak{A}_{v-k} P\right] \leqq v-k+M+k+s\left(P^{-1}\right)
$$

and we define $M_{0}=M+s\left(P^{-1}\right)$. But (5.8) implies that $p\left[\mathfrak{B}_{v}(z)\right] \leqq v+M_{0}$ for all $v=0,1,2, \ldots$ This is contradicted by (5.6) since

$$
v(1+1 / p)>v+M_{0}
$$

when $v>p M_{0}$. Therefore (I) and (II) are incompatible; hence if (1.1) is not regular singular, then there is no fixed constant such that (II) holds for all positive integers $v$.

To conclude our proof, we note that $p\left[\mathfrak{A}_{v}\right] \leqq v q$, since the dominating term of $\mathfrak{A}_{v}$ is $A_{0}^{v} z^{-v q}$. So the condition (1.6) does not impose any restrictions upon the coefficients of the expansion of $\mathfrak{A}_{v}$ unless $v q>v+(n-1)(q-1)$, i.e., $v>n-1$. Therefore conditions (1.6) must be verified for only

$$
v=n, n+1, \ldots, p M_{0}=N
$$

in order to conclude that (1.1) is regular singular.

VI. A bound for $N$ in case $A_{0}$ is nilpotent of full rank. We assume, without loss of generality, that $A_{0}$ is in its Jordan canonical form (4.1), since by a constant similarity transformation we could achieve this and the span of such a transformation is zero, therefore not affecting any of our estimates. Furthermore, we let $T^{*}$ be the transformation

$$
T^{*}=D T
$$


where $D=\operatorname{diag}\left\{1, z^{q}, z^{2 q}, \ldots, z^{(n-1) q}\right\}$, and $T$ is the matrix defined as (4.2), where $A(z)$ now denotes any matrix of the form

$$
A(z)=A_{0} / z^{q}+A_{1} / z^{q-1}+\cdots,
$$

where $A_{0}$ has the form (4.1). It can be verified by a direct computation that $T^{*}(z)$ has the expansion

$$
T^{*}(z)=I+T_{1} z+T_{2} z^{2}+\cdots,
$$

where $T_{1}, T_{2}, \ldots$ are uniquely determined constant matrices, for which the series (6.3) converges elementwise in some neighborhood of 0 . Then obviously $s\left(T^{*}\right)=0$.

Let

$$
Y^{\prime}=\left(T A T^{-1}+T^{\prime} T^{-1}\right) Y=C Y,
$$

where $C(z)=\left(C_{i j}(z)\right)_{1}^{n}$, has the following structure:

(1) $C_{i, i+1}(z)=z^{-q}$ for $i=1,2, \ldots, n-1$,

(2) $C_{i, j}=0$ for all $i>n$ and $j \neq i+1$, and

(3) $C_{n k}(z)$ is some function having a pole of no higher order than $q-1$, $k=1,2, \ldots, n$.

We now wish to reprove the sufficiency of Theorem 2 in such a way as to obtain a bound on both $p$ and $M_{0}$ which arose in the theorem of Turrittin. It should be noted here that the techniques are essentially due to Turrittin, modified to suit our situation.

So we assume that (1.1) is not regular singular. Therefore (6.4) is not regular singular since $Y$ and $X$ are related by $T X=Y$.

If we form the diagonal

$$
\hat{D}=\operatorname{diag}\left\{1, z^{1-q}, z^{2(1-q)}, \ldots, z^{(n-1)(1-q)}\right\}
$$

and make the substitution $Y=\hat{D} U$, then

$$
U^{\prime}=\left(\hat{D}^{-1} C \hat{D}-\hat{D}^{-1} \hat{D}\right) U
$$

can not be regular singular either. But if each function $C_{n k}(z)$ had a zero of at least the order $(n-k)(q-1)-1$, for $k=1,2, \ldots, n$, then the coefficient matrix of the system (6.5) would have a pole of order one, which would violate our assumption. Therefore we may assume that, consistent with our assumption that (1.1) is not regular singular, for some $k, 1 \leqq k \leqq n, C_{n k}(z)$ has a zero of at most the order $(n-k)(q-1)-2$.

We now use a shearing transformation

$$
S(z)=\operatorname{diag}\left\{1, z^{g}, z^{2 g}, \ldots, z^{(n-1) g}\right\},
$$

where $g$ is a real parameter which remains to be determined. 
Then

$S^{-1} C S-S^{-1} S^{\prime}=F=$

$$
\left[\begin{array}{cccccc}
0 & z^{-g-q} & 0 & 0 & \cdots & 0 \\
0 & g z^{-1} & z^{-g-q} & 0 & \cdots & 0 \\
\vdots & & & & & \\
0 & & & 0 & (n-2) g z^{-1} & z^{-g-q} \\
C_{n 1} z^{(n-1) g} & \cdots & C_{n k} z^{(n-k) g} & & \cdots & C_{n n}+(n-1) g z^{-1}
\end{array}\right] .
$$

We seek to select $g$ in (6.6) in such a way that the leading coefficient of $F$, say $F_{0}$, is nonnilpotent. This can be assured by choosing $g$ with the property that at least one term in the $n$th row of $F$ has a series expansion whose leading term has exactly the same order as the,terms on the first super diagonal and no term in the nth row has a leading term of any higher order. Then $F_{0}$ is a companion matrix with at least one term in the $n$th row, therefore not all eigenvalues of $F_{0}$ are zero, and hence $F_{0}$ is nonnilpotent.

If we let $\gamma_{n k}$ denote the order of the zero of $C_{n k}(z)$, then the conditions above may be phrased in terms of the following inequalities: We seek $g=g_{0}$ such that

$$
-q-g_{0}=\gamma_{n k}+(n-k) g_{0} \quad \text { for some } k, 1 \leqq k \leqq n,
$$

while

$$
-q-g_{0} \leqq \gamma_{n, j}+(n-j) g_{0} \quad \text { for all } j, 1 \leqq j \leqq n .
$$

Our restrictions on the functions $C_{n k}(z)$ may be phrased as:

$$
\gamma_{n k} \geqq-q+1
$$

for all $k=1,2, \ldots, n$, and

$$
\gamma_{n k} \leqq(n-k)(q-1)-2
$$

for at least one value $k=k_{0}, 1 \leqq k_{0} \leqq n$.

Since $F=C$ when $g=0$, the inequalities (6.8) are satisfied initially due to the condition (6.9). We then make $g$ decrease until we attain the first critical value $g_{0}$ for which the equality (6.7) holds. Then

$$
g_{0}=\frac{-q-\gamma_{n k}}{n-k+1} \geqq \frac{-q-\gamma_{n j}}{n-j+1}, \quad 1 \leqq j \leqq n .
$$

Therefore

$$
-g_{0} \leqq q-\frac{n-k+2}{n-k+1}
$$

due to (6.10), and hence the leading term in the expansion of $F$ is $F_{0} z^{-q-g_{0}}$, where

$$
q+g_{0} \geqq 1+1 / n \text {. }
$$


If $\left\{\mathfrak{F}_{v}\right\}$ denotes the sequence associated with $F$ as in Definition (1.4), then from (6.12) we have

$$
p\left[\mathfrak{F}_{v}\right]=v\left(q+g_{0}\right) \geqq v(1+1 / n) .
$$

Furthermore, $s(S(z))=-g_{0}(n-1)$, therefore

$$
s(S(z)) \leqq(q-1-1 / n)(n-1)=(n-1) q-n+1 / n .
$$

Just as in the proof of Theorem 2, we again have the relations:

$$
\mathfrak{F}_{v}=\sum_{k=0}^{v}\left(\begin{array}{l}
v \\
k
\end{array}\right)\left(S^{-1}\right)^{(k)} C_{v-k} S, \quad v=0,1,2, \ldots
$$

Since $C$ and $A$ are related as in (6.4) by a transformation whose span is zero, if we assume (1.6), then

$$
p\left[C_{v}\right] \leqq v+(n-1)(q-1)
$$

for all $v$ also. Now using our bound (6.14) on the span of $S$ (and hence of $S^{-1}$ ), we apply the result of Lemma 3 to conclude that

$$
p\left[\left(S^{-1}\right)^{(k)} C_{v-k} S\right] \leqq k+v-k+(n-1)(q-1)+(n-1) q-n+1 / n .
$$

Therefore $p\left[\mathfrak{F}_{v}\right] \leqq v+2(n-1) q-2 n+1+1 / n$.

But from (6.13), we see that

$$
v(1+1 / n)>v+2(n-1) q-2 n+1+1 / n
$$

when $v \geqq 2 n^{2}(q-1)+n(2 q-1)$. Therefore if (1.1) is not regular singular, condition (1.6) will be violated for at least one $v$,

$$
v=n, n+1, \ldots, 2 n^{2}(q-1)+n(2 q+1) .
$$

Hence for sufficiency of the condition in Theorem 2, we need only check (1.6) for these values of $v$.

The sharpness of this bound is not claimed and, in fact, it is felt by the author that this bound is much too generous. The result however, does complete Jurkat's theorem to a form which is now aesthetically more pleasing, at least in this special case. When the leading coefficient matrix has a more general structure, then the situation necessarily becomes more complicated.

VII. A further application of Lemma 1. In his work on the problem, Horn showed that for each system (1.1) which is regular singular, there exists $T \in H(A)$ such that

$$
T(z)=\sum_{v=-M}^{M} T_{v} z^{v}
$$

however no bounds on $M$ were given. Lately, J. Moser has asked [5, p. 380] if it is possible to tell, in advance of constructing the transformation for a given system, how many terms in the expansion are required. We give here an upper bound on the number of terms which are necessary. 
If (1.1) is regular singular, there exists $T \in H(A)$, with $T$ having the Laurent expansion (7.1), in which $M=n(q-1)$.

Proof. From Lemma 1 we have $T^{*} \in H(A), T^{*}=P D$, where $P$ is a unit in $\mathscr{R}$, $D$ is a diagonal having $\operatorname{span} s(D) \leqq(n-1)(q-1)$, and $D \in H(\hat{A})$.

We first seek to replace $P$ by a polynomial truncation $P_{(m)}$ after the $m$ th term in the expansion. Let

$$
\hat{A}_{(m)}=P_{(m)}^{-1} A P_{(m)}-P_{(m)}^{-1} P_{(m)}
$$

We must choose $m$ so large that $D \in H\left(\hat{A}_{(m)}\right)$, where the only information we have about $D$ is $s(D) \leqq(n-1)(q-1)$.

If $P=\sum_{v=0}^{\infty} P_{v} z^{v}$, then $P^{-1}=\sum_{v=0}^{\infty} P_{v}^{*} z^{v}$, where $P_{v}^{*}$ depends only upon $P_{0}$, $P_{1}, \ldots, P_{v}$. This is seen by investigating the system of matrix equations which define the coefficients of $P^{-1}$.

Therefore if we let $\hat{A}-\hat{A}_{(m)}=C_{(m)}$, then $C_{(m)}$ has a zero of at least the order $m-q$. Then $D^{-1} C_{(m)} D$ has, according to Lemma 2, a pole whose order does not exceed $(n-1)(q-1)-(m-q)$. If $(m-q)-(n-1)(q-1) \geqq-1$, i.e., if $m \geqq n(q-1)$, then $D \in H\left(\hat{A}_{(m)}\right)$, hence

$$
T=P_{((n-1) q)} D \in H(A) .
$$

We are still free to choose $\alpha_{n}=-(q-1)$ if we wish. This means that $\alpha_{1} \geqq n(1-q)$, therefore

$$
D=\sum_{v=n(1-q)}^{-q+1} D_{v} z^{v} .
$$

Then $T$, defined as in (7.3), has the expansion (7.1) with $M$ replaced by $n(q-1)$.

\section{REFERENCES}

1. Philip Hartman, Ordinary differential equations, Wiley, New York, 1964; Chapter IV.

2. Donald A. Lutz, On systems of linear differential equations having regular singular solutions, Journal of Differential Equations (to appear).

3. J. Horn, Zur Theorie der Systeme linearer Differentialgleichungen mit einer unabhängigen Verändlichen. II, Math. Ann. 40 (1892), 527-550.

4. C. C. MacDuffee, The theory of matrices, Chelsea, New York, 1933.

5. J. Moser, The order of a singularity in Fuchs' theory, Math. Z. 72 (1960), 379-398.

6. H. L. Turrittin, Convergent solutions of ordinary homogeneous differential equations in the neighborhood of an irregular singular point, Acta Math. 93 (1955), 27-66.

UNIVERSITY OF WISCONSIN, MilwaUKeE, WisCONSIN 